\title{
Status of Health Information Classification for Consumer Information Retrieval
}

\author{
Melinda Whetstone \\ Doctoral student \\ College of Information, Florida State University \\ Tallahassee, FL 32301 \\ Email: mrw06j@fsu.edu
}

With a growing movement toward patient-centric health care and patient empowerment, consumers are encouraged to take an active role in their health care. In fact, nearly $80 \%$ of Americans are taking to the Internet to search for health information (Fox, 2006). In addition to the Internet, consumers have access to a growing number of health search engines and professional biomedical databases via health science libraries. However, the consumer's ability to efficiently retrieve information that is understandable and pertinent to their query is hindered (Volk, 2007) for reasons that include inexperience with "technical terminology," (Zeng \& Tse, 2006) and poor database usability (Smith, 2007).

Several organizations are engaged in efforts to refine capabilities to match consumer health queries with accurate information (Smith, 2007; Zeng \& Tse, 2006; Zeng et al., 2006).

However, as more consumers seek health information in this growing empowered climate and more information is made available, there is an increasing danger that the consumer will become more confused and less able to find pertinent information. This paper explores three areas: it examines the current biomedical ontologies that are used for consumer health searches; it looks at the current means for health information retrieval (IR); and it explores ongoing projects that serve to improve consumer search capabilities. To evaluate the effects of multiple ontologies and search methods, a consumer's health query was posed using several health search methods.

\section{Current Search Methods}

Currently, many avenues exist in which consumers may search for information, including general Internet search engines (e.g. Google and Yahoo), specific Internet health search engines (e.g. Healthline and Healia), online medical sites (e.g. Merck and WebMD), professional databases provided via libraries (e.g. Thomson \& Gale Health and Wellness Resource Center), clinical databases (e.g. Medline and CINAHL), and health lists provided by professional organizations, e.g. Medical Library Association. These products offer differing means for information organization, i.e. vertical hierarchical classification (Google, 2006), and retrieval, i.e. full-text, concept-based, and context sensitive, that affect ease of use and retrieval success.

\section{A Consumer's Health Query Review}

Several search tools available for health queries were evaluated for their effectiveness in finding material pertaining to the specific query <depression treatment>. The databases selected for evaluation were: 1) CINAHL, 2) Thomson Gale Health \& Wellness Resource Center, 3) Medline Plus, 4) Google, 5) Healia, and 6) WebMD. Results include general findings that all tools provide means for query refinement and that web tools return a greater number of search results.

Possible future directions:

The optimal consumer IR tool would satisfy two primary criteria. First, it would be sensitive to the individual and their information needs, e.g. the consumer's literacy levels and the required 
level of detail (e.g. general information versus protocol specific information). Secondly, it would be a one-stop tool that would draw upon all known bodies of information. Future direction for research and efforts to satisfy these criteria will be discussed.

\section{References}

Fox, S. (2006). Online Health Search 2006. Washington, DC: Pew Internet \& American Life Project.

Smith, C. A. (2007). The Ten Thousand Questions project: Towards a better understanding of consumer health vocabulary. Journal of Consumer Health on the Internet, 11(1), 33-47.

Volk, R. M. (2007). Expert searching in consumer health: an important role for librarians in the age of the Internet and the Web. Journal of the Medical Library Association, 95(2), 203-207.

Zeng, Q. T., Crowell, J., Plovnick, R. M., Kim, E., Ngo, L., \& Dibble, E. (2006). Assisting Consumer Health Information Retrieval with Query Recommendations. Journal of American Medical Information Association, 13, 80-90.

Zeng, Q. T., \& Tse, T. (2006). Exploring and developing consumer health vocabularies. Journal of American Medical Information Association, 13, 24-29. 